\title{
ESTUDO ELETROMIOGRÁFICO DA DEGLUTIÇÃO NA MUSCULATURA SUPRA-HIÓIDEA EM SUJEITOS CLASSE I E III DE ANGLE
}

\author{
Electromyographic study of swallowing in \\ suprahyoid musculature in Angle's Class I and III subjects
}

\author{
Mirian Hideko Nagae ${ }^{(1)}$, Marcelo Corrêa Alves ${ }^{(2)}$
}

\begin{abstract}
RESUMO
Objetivo: comparar a atividade elétrica do músculo supra-hióideo durante a deglutição em sujeitos com distintos padrões morfológicos e dentários. Métodos: foram realizados registros eletromiográficos de superfície em 32 voluntários saudáveis e sem Disfunção Temporomandibular, subdivididos em dois grupos, 16 sujeitos Classes I e 16 Classe III de Angle, gênero masculino e feminino, com faixa etária entre 18 e 25 anos de idade. Resultados: foram obtidos os valores do Root Mens Square ( $p<$ $0,05)$ : original Classe I $(15,238)$ e Classe III $(32,550)$; normalizado pelo pico máximo Classe I $(29,765)$ e Classe III $(42,094)$; normalizado pela média Classe I $(29,332)$ e Classe III $(42,327)$. Período ativo do ciclo $(p<0,05)$ Classe I $(29,7648)$ e Classe III $(42,0937)$; instante de máxima atividade $(p>0,05)$ Classe I $(47,6485)$ e Classe III $(47,9437)$. Conclusão: os sujeitos Classe I demonstraram uma amplitude média menor, com aumento e diminuição da atividade elétrica sincrônica e equilibrada. Os sujeitos Classe III apresentaram uma amplitude média e um período ativo maior, assincrônico e desequilibrado durante a deglutição.
\end{abstract}

DESCRITORES: Deglutição; Má Oclusão de Angle Classe III; Eletromiografia

\section{INTRODUÇÃO}

As implicações de padrões morfológicos dentoesqueletais distintos têm sido vastamente estudadas sob vários aspectos, como área de contato oclusal ${ }^{1,2}$, ritmo mastigatório ${ }^{3,4}$, e posicionamento do bolo alimentar na cavidade bucal ${ }^{5}$.

A musculatura, além de delimitadora das partes da cavidade bucal é agente determinante de sua mobilidade. Estudos relatam sua importância desde a fase de crescimento crânio-facial, por exercerem influência significativa na conformação óssea ${ }^{4-6}$.

(1) Fonoaudióloga; Professora de Motricidade Orofacial do curso de Fonoaudiologia da Universidade Estadual de Campinas, UNICAMP, Campinas, SP; Mestre e Doutora em Biologia Buco-Dental pela Faculdade de Odontologia de Piracicaba da Universidade Estadual de Campinas.

(2) Analista de sistemas; Professor da Faculdade Anhanguera de Piracicaba, Piracicaba SP; Doutorando em Biologia Buco-Dental pela Faculdade de Odontologia de Piracicaba da Universidade Estadual de Campinas.

Conflito de interesses: inexistente
Uma ação conjunta e equilibrada das estruturas maxilo-mandibular com o sistema muscular é de suma importância para que funções, como: mastigação ${ }^{7}$, deglutição, fala e respiração, ocorram de forma eficiente ${ }^{8}$

Em casos de desvios crânio-faciais e/ou oclusais é esperado que a plasticidade dos tecidos moles favoreça mecanismos de adaptação ${ }^{9-11}$, dentre os quais se destaca o das compensações musculares ${ }^{12}$ que buscam o equilíbrio do sistema estomatognático para a execução das tarefas. A compreensão de como a musculatura atua nesses sujeitos, no entanto, ainda é pouco conhecida ${ }^{13,14}$.

Dinâmicas musculares em indivíduos com discrepâncias esqueletais como prognatismo mandibular ilustram claramente tais situações. Eventos significativos na biomecânica em dinâmicas, como a deglutição ${ }^{15}$, podem necessitar de ajustes para que o sistema estomatognático mantenha-se estável e a função ocorra ${ }^{16}$.

O músculo supra-hióideo é considerado estratégico na deglutição, pois participa de mecanismos 
motores reflexos ${ }^{17,18}$, do abaixamento e estabilização mandibular ${ }^{19}$, elevação e anteriorização do osso hioide ${ }^{13,14,17,18}$ e possui inserções conjuntas à musculatura da língua, a qual é intensamente recrutada nos sujeitos prognatas ${ }^{20}$.

Como uma das preconizações fonoaudiológicas é a interveção mioterápica, a compreensão do comportamento muscular é de suma importância para a avaliação, diagnóstico ${ }^{21}$ planejamento terapêutico, desenvolvimento de técnicas ${ }^{22}$, prognóstico e compreensão de recidivas do tratamento ${ }^{23,24}$.

Isto é particularmente importante no caso de desvios, tais como o prognatismo mandibular. Relatos de casos na literatura envolvendo a eletromiografia de superfície na musculatura supra-hióidea ${ }^{25-27}$ são escassos e com resultados variados ${ }^{25,27}$.

Este trabalho tem como objetivo a investigação do comportamento da musculatura supra-hióidea durante a deglutição por meio da eletromiografia de superfície em sujeitos Classe I e III de Angle.

\section{MÉTODOS}

A amostra foi composta por 16 sujeitos Classe I e 16 Classe III de Angle, gênero masculino e feminino com faixa etária entre 18 e 30 anos, oriundos dos quadros de discentes e funcionários da instituição. Não apresentavam disfunção temporomandibular, ausência de elementos dentais, dor muscular, uso de medicamentos miorrelaxantes e não foram submetidos a tratamento ortodôntico e/ou ortopédicos. O experimento foi realizado de acordo com as normas estabelecidas pela International Society of Electromyography and Kinesiology (ISEK) ${ }^{28}$. Os voluntários, após lerem e ter esclarecido os procedimentos aos quais se submeteriam, assinaram o Termo de Consentimento Livre e Esclarecido (TCLE). Em seguida foi realizada anamnese, avaliação clínica e odontológica pela pesquisadora e por uma dentista respectivamente.

\section{Registro Eletromiográfico}

Previamente à fixação dos eletrodos, os voluntários realizaram, quando necessário, tricotomia e em seguida, limparam a pele com álcool etílico. 0 registro foi realizado com os sujeitos sentados e a cabeça posicionada paralela ao plano de Frankfurt.

O equipamento utilizado foi o eletromiógrafo Myosystem I acoplado a um computador Pentium ${ }^{\circledR} 4$ (Intel) de 650 Mhz e software Myosystem BR, versão 2.52 (DataHominis Tecnologia Ltda). Condicionador de sinal com 12 bits de resolução e CMRR (Modo de Rejeição Comum) de 112dB @ $60 \mathrm{~Hz}$. O sinal eletromiográfico foi obtido com um conversor Analógico Digital (A/D) Myosystem (Prosecon Ltda), modelo PCI-DAS 1200 com 12 bits de resolução.
A frequência de amostragem foi de $2000 \mathrm{~Hz}$ e a atividade elétrica foi detectada com eletrodos bipolares descartáveis de $\mathrm{Ag} / \mathrm{AgCl}$ modelo Chicopee MA011 (Meditrace $®$, Kendall-LTP) com $1 \mathrm{~cm}$ de diâmetro, acoplado a um pré-amplificador, modelo PA 1010-VA (LynxTecnologia Eletrônica Ltda), com 20 vezes de ganho, formando o circuito de um eletrodo diferencial. $O$ eletrodo de referência foi posicionado junto ao osso esterno do voluntário e o do músculo supra-hióideo posicionados na linha média abaixo do queixo, seguindo em direção ântero-posterior ${ }^{29}$. Foi realizado teste de função ${ }^{30}$, palpando-se a área embaixo do queixo e solicitando que o voluntário deglutisse. Com base na identificação do posicionamento do músculo, fixou-se o eletrodo em seu ventre muscular ${ }^{29}$.

A distância intereletrodo foi de $1 \mathrm{~cm}$ e a amplificação do sinal foi realizada de maneira a ocupar 2/3 da tela com a finalidade de se obter a melhor resolução do sinal. Os voluntários mantinham $5 \mathrm{ml}$ de água na boca ${ }^{18,31}$ e após ordem verbal deglutiam o líquido. A capitação dos registros foi realizada num período de 5 segundos.

\section{Análise do processamento}

Os registros eletromiográficos foram submetidos a um filtro Butterworth passa alta de $20 \mathrm{~Hz}$ e passa baixa de $500 \mathrm{~Hz}$, onde foram calculados os valores do Root Means Square (RMS) ${ }^{32}$ a fim de se representar a amplitude média do sinal eletromiográfico, conforme fórmula abaixo:

$$
R M S=\sqrt{\frac{1}{N} \sum_{i=1}^{n} x_{i}^{2}}
$$

Em que " $N$ " representa o número de pontos medidos no período e " $x$ " representa a diferença média no potencial observado $(\mu \mathrm{V})$.

$\mathrm{Na}$ eletromiografia cuidados como a normalização, a qual permite que possíveis variabilidades nos procedimentos ${ }^{32}$ e diferenças interindividuais entre os sujeitos e grupos possam ser atenuados, devem ser realizados ${ }^{32,33}$. Critérios inerentes ao próprio sujeito nesse caso são utilizados para a obtenção de um perfil homogêneo da amostra ${ }^{32}$.

Após a obtenção do RMS original, os sinais foram normalizados por meio do software MATLAB, segundo o tempo, retificados e submetidos a um filtro passa baixo de $4 \mathrm{~Hz}$. Por fim, os ciclos foram sobrepostos e foi calculada a média ao longo do tempo. Os dados médios foram normalizados no domínio da amplitude de acordo com dois critérios (pico e média) tendo sido selecionado o pico como critério mais apropriado para representação dos dados, visto que este apresentou menor 
Coeficiente de Variação (CV) ${ }^{30}$, conforme apresentado na Tabela 1.

Após a normalização dos dados, variáveis que identificavam o período de ativação médio dos ciclos (ON) e o instante de máxima ativação (IMAX) foram analisados.

Este trabalho foi submetido e aprovado pelo Comitê de Ética em Pesquisa (CEP) da Faculdade de Odontologia de Piracicaba da Universidade Estadual de Campinas-FOP/UNICAMP (processo $n^{\circ}$ 188/2003).

\section{Análise estatística}

Os dados foram testados para a existência de homogeneidade de variância nos grupos, presença de valores discrepantes, existência de dados excessivamente influentes e para a escala da variável de resposta através do software SAS/LAB ${ }^{34}$.
Esta análise mostrou problemas com a escala das cinco variáveis de resposta (RMS original, RMS normalizado pelo pico, RMS normalizado pela média, instante de máxima ativação - IMAX e tempo de ativação nos ciclos - ON) e para mitigar este problema, um estudo de transformação para a potência ótima sugeriu que a aplicação da função logaritmo resultaria em valores mais facilmente modelados em todas as variáveis.

Uma vez aplicada à transformação e não havendo outros sinais de problemas com as suposições que embasam a técnica adotada, os dados foram submetidos à análise de variância com um fator, o que permitiu fazer inferências sobre a existência de diferenças entre as médias verdadeiras de RMS nos grupos (Classe I e Classe III de Angle).

Foi adotado um nível de significância de 5\% para conclusões neste estudo.

Tabela 1 - Coeficiente de variação dos valores de RMS normalizado segundo os critérios do pico máximo e média

\begin{tabular}{lcc}
\hline & CV (pico máximo) & CV (média) \\
\hline CLASSE I & $71,3 \%$ & $71,8 \%$ \\
CLASSE III & $77,2 \%$ & $80,5 \%$ \\
\hline
\end{tabular}

\section{RESULTADOS}

A observação do comportamento médio de todos os voluntários é apresentada na Figura 1, na qual se observa uma menor variabilidade nos sujeitos
Classe I (CV: 71,3\%) e nos sujeitos Classe III (CV: $77,20 \%$ ) com a utilização do critério pico máximo.

Uma vez avaliados os valores da amplitude, passa-se à análise dos valores de RMS através da análise de variância apresentada na Tabela 2.
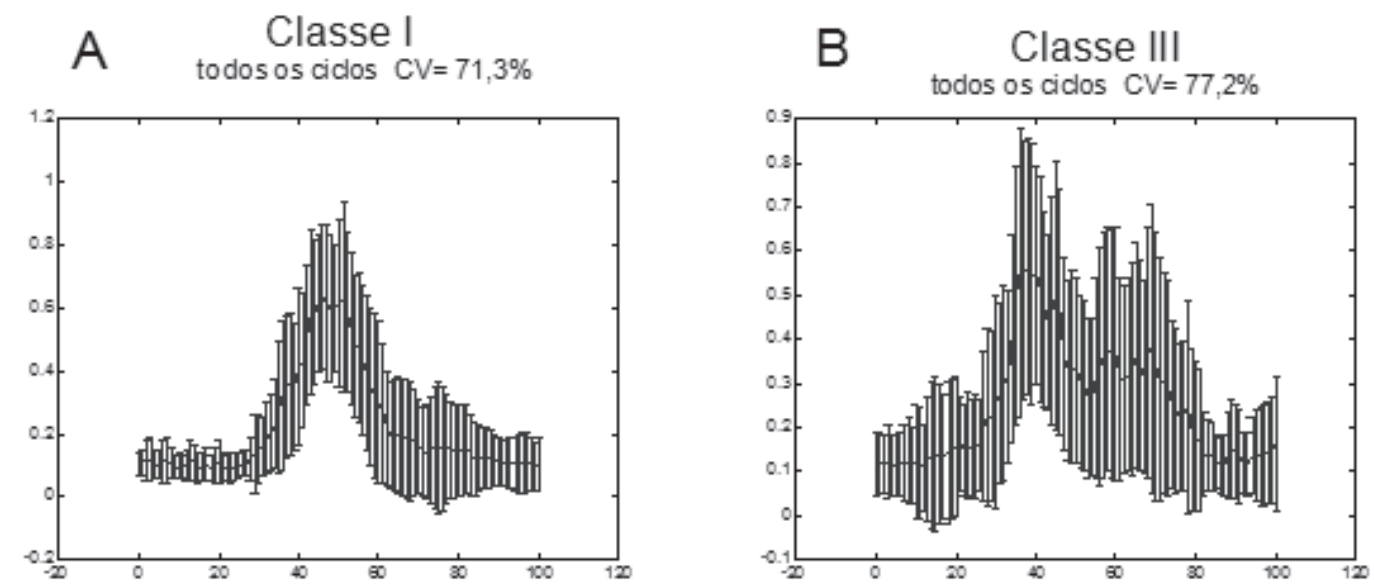

Figura 1 - Representação da amplitude $(\mu \mathrm{V})$ do ciclo médio de todos os sujeitos sobrepostos formando um único ciclo observado através da eletromiografia. Músculo supra-hióideo, normalizado pelo pico máximo nos sujeitos Classe I (A) e Classe III (B) de Angle. Observar maior amplitude nos sujeitos Classe III que os sujeitos Classe I o que denota uma situação mais constante nos sujeitos Classe I que nos Classe III, tanto no período de repouso quanto no período ativo 
Tabela 2 - Quadro de análise de variância para experimentos com um fator para análise da variável RMS original, normalizada pelo pico e normalizada pela média, instante de máxima ativação e tempo de ativação transformada pela função logaritmo, de acordo com estudo preliminar de suposições

\begin{tabular}{lcccc}
\hline Variáveis & $\begin{array}{c}\text { Quadrados } \\
\text { médios de } \\
\text { grupos }\end{array}$ & $\begin{array}{c}\text { Quadrados } \\
\text { médios do } \\
\text { resíduo }\end{array}$ & Estatística F & Valor-p \\
\hline RMS original & 0,9273 & 0,6591 & 42,21 & $<0,0001$ \\
RMS normalizado pela média & 0,2030 & 0,0370 & 5,48 & 0,0261 \\
RMS normalizado pelo pico & 0,2122 & 0,0412 & 5,15 & 0,0305 \\
Instante de Máxima ativação & 0,0042 & 0,0470 & 0,09 & 0,7665 \\
Tempo de ativação & 1,1251 & 0,2183 & 5,15 & 0,0305 \\
\hline
\end{tabular}

Esta análise é usada para detectar diferença estatística entre as médias verdadeiras das variáveis de resposta, transformadas de acordo com a função logaritmo nos diferentes níveis do fator Classe.

São observados fortes índicos $(p<0,01)$ da existência de diferenças entre as médias de RMS nas Classes, quando não há normalização dos dados, conforme ilustra a Figura 2, que permite concluir pela maior média $(32,550)$ no grupo Classe III em relação à média $(15,238)$ do grupo composto por pessoas Classe I de Angle.

Conclusões similares são observadas quando analisados os valores normalizados pela média e pelo pico, entretanto, a estatística dá indícios $(p<0,05)$ da existência de diferenças entre as médias verdadeiras $(p<0,05)$ de RMS dos grupos Classe I e Classe III, mesmo quando a normalização suprime as diferenças interpessoais de acordo com os dois critérios testados.
Quando normalizados pelas médias e pelo pico, a média de RMS ainda aponta uma maior atividade no grupo Classe III, entretanto, as diferenças diminuem em relação ao observado nos dados originais (sem normalização) conforme ilustra a Figura 3.

Podem ser observadas sobreposições nos intervalos de confiança da média dos grupos Classe I e III em cada condição de normalização, diferente do que ocorre nos dados brutos o que evidencia uma supressão de diferenças decorrentes da normalização, fator desejado quando se suprimem as diferenças interpessoais.

A análise do IMAX não nos dá indícios da existência de diferenças entre as médias ( $p>0,90)$, sendo assim, não se pode inferir que existem diferenças entre as médias desta variável nos grupos estudados, conforme ilustra a Figura 4.

A análise de variância nos dá indícios $(p<0,05)$ da existência de diferenças entre as médias verdadeiras do ON nos diferentes níveis do fator Classe, conforme ilustra a Figura 5.

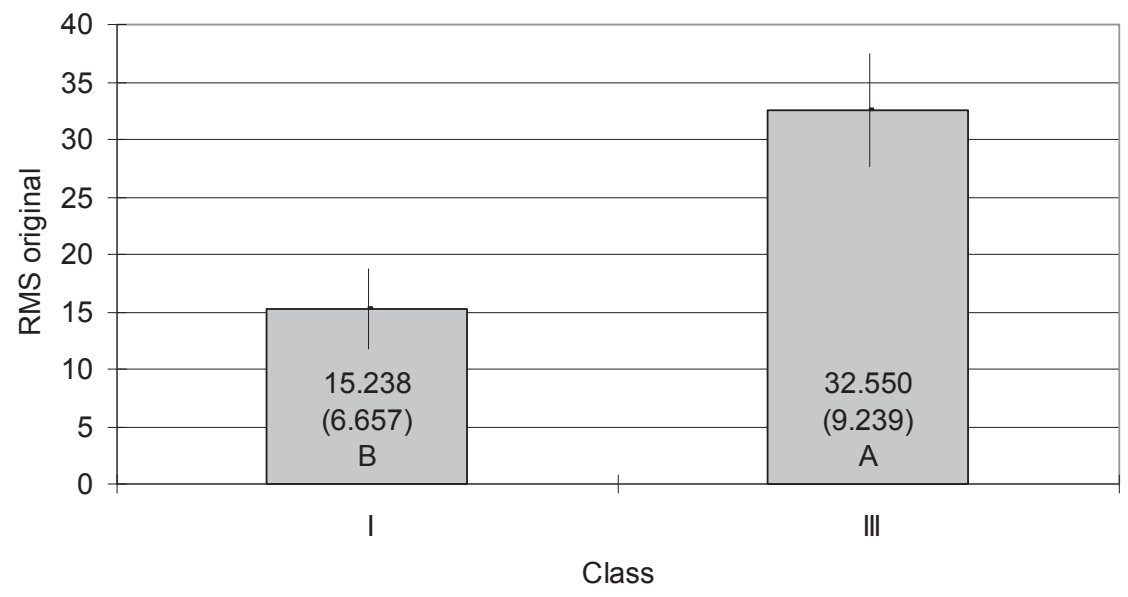

Figura 2 - Média (desvio padrão) e limites do intervalo de confiança da média (95\%) dos valores de RMS original. Médias com letras iguais não diferem entre si pela análise de variância com nível de significância de $5 \%$ 

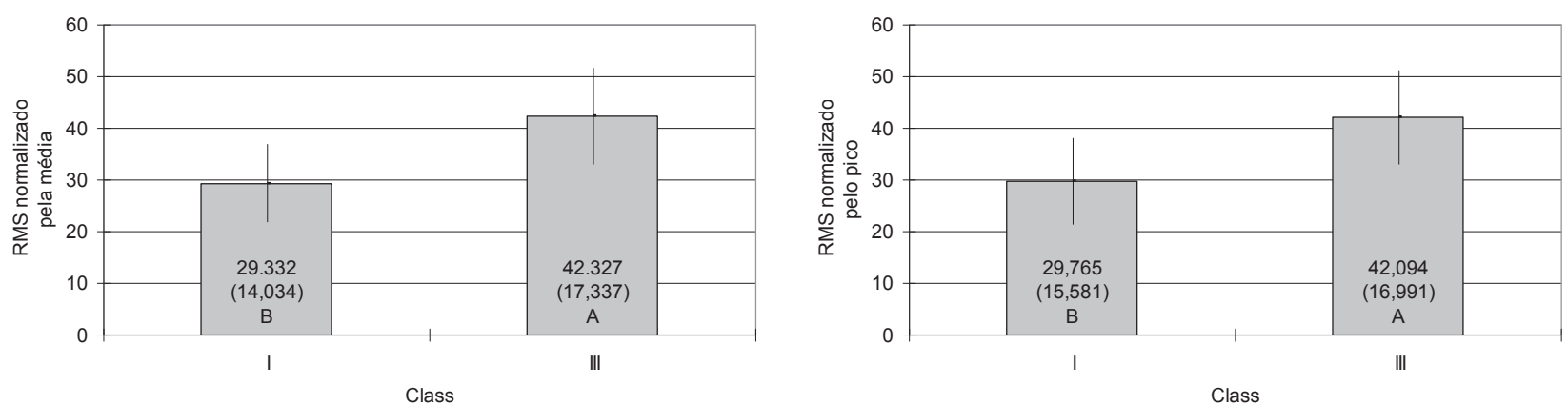

Figura 3 - Média (desvio padrão) e limites do intervalo de confiança da média (95\%) dos valores de RMS normalizados a média (A) e pelo pico (B). Médias com letras iguais não diferem entre si pela análise de variância com nível de significância de $5 \%$

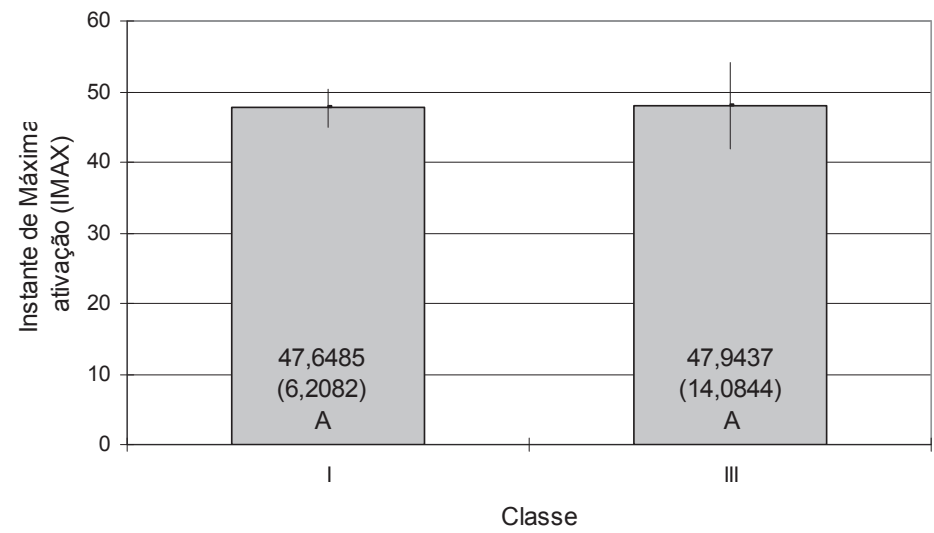

Figura 4 - Média (desvio padrão) e limites do intervalo de confiança da média (95\%) dos valores de Instante de máxima ativação. Médias com letras iguais não diferem entre si pela análise de variância com nível de significância de 5\%

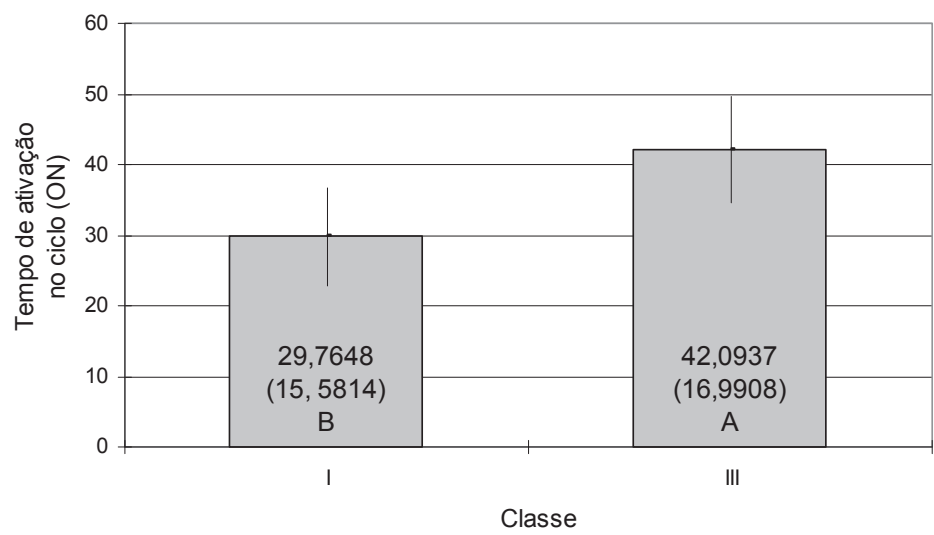

Figura 5 - Média (desvio padrão) e limites do intervalo de confiança da média (95\%) dos valores tempo de ativação no ciclo. Médias com letras iguais não diferem entre si pela análise de variância com nível de significância de 5\%

\section{DISCUSSÃO}

A deglutição compreende o ato de engolir, realizada pelas fases oral, preparatória, faríngea e esofágica, cujas dinâmicas envolvem estruturas anatômicas específicas. A sistematicidade e frequência da deglutição de 300 a 2400 vezes ao dia ${ }^{35}$ envolvem uma coordenação precisa do 
sistema estomatognático e das estruturas que o compõe. Ao comparar os registros eletromiográficos durante a deglutição no músculo supra-hióideo pôde-se constatar um padrão de comportamento com início e finalização claramente demarcado, porém com formas distintas de atuação entre os sujeitos Classe I e III de Angle.

$\mathrm{Na}$ análise qualitativa dos sujeitos Classe I de Angle houve um aumento e diminuição gradativo da atividade de forma equilibrada, já nos sujeitos Classe III o padrão de atividade foi inconstante e assincrrônico (Figura 1). Para visualizar quantitativamente os dados colhidos, valores do RMS foram utilizados para investigação da amplitude dos sinais. Nos sujeitos Classe I os dados obtidos de RMS foram menores $(15,238)$ do que nos sujeitos Classe III $(32,550)$ explicitando diferenças entre as médias nas Classes $(p<0,05)$. Comprovando com isso a importância de um padrão morfológico e oclusal adequados a fim de que a musculatura não necessite exercer sobrecargas e a estabilidade do sistema estomatognático seja mantida ${ }^{36,37}$.

O maior RMS obtido nos sujeitos Classe III, demonstra a necessidade de um maior esforço muscular decorrentes provavelmente da desproporção maxilo-mandibular e do tipo de encaixe das chaves de oclusão ${ }^{36}$. A protrusão mandibular isolada sem alteração oclusal, no entanto, não é suficiente para ocasionar uma atividade eletromiográfica diferenciada ${ }^{19}$. A conjugação de fatores morfológicos distintos, como maxila, mandíbula e oclusão, é necessária a fim de que a musculatura atue com outro padrão, como se pôde constatar no presente estudo. Nos sujeitos Classe III o posicionamento mandibular também favorece uma reorganização de outras estruturas como a língua ${ }^{20}$ a qual ao se alojar no assoalho bucal favorece um estado de flacidez muscular e a necessidade de um maior abaixamento mandibular e recrutamento de força para que a deglutição possa ocorrer.

Após a análise qualitativa e do RMS, os dados foram submetidos a normalização para obter uma amostra homogênea em que diferenças de procedimentos, como posicionamento de eletrodos e interdindividuais como presença de tecido adiposo, fossem atenuados ${ }^{33}$. As amostras foram submetidas aos critérios de pico máximo e média do sinal eletromiográfico, em que análises estatísticas demonstraram que após a normalização houve diminuição de diferença entre as classes, uma vez que os dados foram suprimidos para a atenuação das diferenças. Mesmo com a diminuição, diferenças estatísticas entre as classes foram constatadas com predomínio da atividade nos sujeitos Classe III tanto no pico máximo $(42,094)$ como na média $(42,327)$ em relação aos sujeitos Classe I que apresentaram valores menores de pico máximo $(29,765)$ e média $(29,332)$. A submissão dos dados ao pico máximo e média permite detectar também qual critério torna a amostra mais homogênea, a partir do cálculo do menor CV. O menor valor obtido (Tabela 1) foi o do pico máximo, $71,3 \%$ para o sujeitos Classe le e $77,2 \%$ para os Classe III, sendo, portanto, este o critério utilizado. A partir do critério escolhido, outras variáveis, como período ativo do ciclo (ON) e instante de máxima atividade (IMAX), foram analisadas.

A investigação do IMAX não demonstrou diferenças estatisticamente significantes entre a Classes I $(47,6485)$ e Classe III $(47,937)$ (Figura 4), provavelmente devido à supressão dos dados decorrente da normalização. Apesar da implementação da normalização exigida como rotina nos trabalhos eletromiográficos, os estudos ${ }^{33} \mathrm{em}$ sua maioria são voltados para músculos de grosso calibre ${ }^{27,38}$ onde a amplitude dos sinais é intensa e claramente caracterizada. Músculos, no entanto, como os da região da cabeça e pescoço são em sua maioria de baixo calibre, cujas diferenças decorrentes de movimentações distintas, as quais se desejam caracterizar, podem desaparecer aos normalizar os sinais.

O maior período ativo (ON) de atividade obtido nos sujeitos Classe III $(42,0937)$ em relação aos sujeitos Classe I $(29,7648),(p<0,05)$ no estudo (Figura 5) comprova os trabalhos ${ }^{39}$ que referem que a modulação do comando motor é dada pela cinestesia da mandíbula e língua, as quais são intensas nos sujeitos prognatas.

A existência de comandos moduladores motoneurais e de estruturas como a musculatura, osso e dentes justificam a diferenciação de padrões de atividades elétricas em uma mesma situação, porém de grupos distintos morfológicos ${ }^{26}$ como os observados no estudo. Além dos comandos sejam eles centrais ou periféricos, há uma engrenagem biomecânica a ser considerada na qual diante da conformação óssea e dental estabelecida, a plasticidade do tecido mole muscular pode ser recrutada como forma de manutenção do equilíbrio do sistema estomatognático a fim de que as funções possam ocorrer.

\section{CONCLUSÃO}

Desta maneira pode-se concluir que o músculo supra-hióideo durante a deglutição apresenta um comportamento diferenciado nos sujeitos Classe I e III de Angle. 


\begin{abstract}
Purpose: to compare Suprahyoid musculature's electric activity during swallowing in subjects with dissimilar morphological and dental patterns. Methods: surface Electromyography's records were taken from 32 healthy volunteers who do not have Temporomandibular Dysfunction, subdivided into two groups, Angle's 16 Class I subjects and 16 Class III subjects, males and females, aging from 18 to 25-year old. Results: the values to Root Mens Square $(p<0.05)$ : original Class I (15.238) e Class III (32.550); normalized by maximum peak Class I (29.765) e Class III (42.094); normalized by medium Class I (29.332) e Class III (42.327). Cycle's active period $(p<0.05)$ Class I (29.7648) e Class III (42.0937); maximum activity instance ( $p>0.05$ ) Class I (47.6485) e Class III (47.9437). Conclusion: class I subjects showed a smaller average amplitude, with increase and decrease of synchronized and balanced electric activity. Class III subjects showed a larger average amplitude and a larger desynchronized and unbalanced period during deglutition.
\end{abstract}

KEYWORDS: Deglutition; Malocclusion, Angle Class III; Electromyography

\section{REFERÊNCIAS}

1. Kobayashi T, Honma K, Nakajima T, Hanada $\mathrm{K}$. Masticatory function in patients with mandibular prognathism before and after orthognathic surgery. J Oral Maxillofac Surg. 1993; 51(9):997-1001.

2. Ferrario VF, Tartaglia GM, Galletta A, Grassi GP, Sforza $C$. The influence of occlusion on jaw and neck muscle activity: a surface EMG study in healthy young adults. J Oral Rehabil. 2007; 33(5):341-8.

3. Maeda K, Ono T, Otsuka R, Ishiwata Y, Kuroda $\mathrm{T}$, Ohyama K. Modulation of voluntary swallowing by visual inputs in humans. Dysphagia. 2004; 19(1):1-6.

4. Takeda $H$, Nakamura $Y$, Handa $H$, Ishii $H$, Hamada Y, Seto K. Examination of masticatory movement and rhythm before and after surgical orthodontics in skeletal Class III patients with unilateral posterior cross-bite. J Oral Maxillofac Surg. 2009; 67(9):1844-9.

5. Lunderberg M, Nord PG, Astrand P. Changes in masticatory function after surgical treatment of mandibular prognathism: cineradiographic study of bolus position. Acta Odontol Scand. 1974; 32:39-49. 6. Moss ML. The primacy of functional matrices in orofacial growth. Dent Pract Dent Rec. 1968; 19(2):65-73.

7. Hanawa S, Tsuboi A, Watanabe M, Sasaki K. EMG study for perioral facial muscles function during mastication. J Oral Rehabil. 2008; 35(3):159-70.

8. Moss JP, Chalmers CP. An electromyographic investigation of patients with a normal jaw relationship and a Class III jaw relationship. Am J Orthod. 1974; 66(5):538-56.

9. Souza DR, Semeghini TA, Kröll LB, Berzin F. Oral myofunctional and electromyographic evaluation of the anterior suprahyoid muscles and tongue thrust in patients with Class II/1 malocclusion submitted to first premolar extraction. J Appl Oral Sci. 2007; 15(1):24-8.

10. Souza DR, Semeghini TA, Kröll LB, Berzin F. Oral myofunctional and electromyographic evaluation of the orbicularis oris and mentalis muscles in patients with Class II/ 1 malocclusion submitted to first premolar extraction. J Appl Oral Sci. 2008; 16(3):226-31.

11. Sabashi K, Saitoh I, Hayasaki H, Iwase $Y$, Kondo S, Inada E, et al. A cross-sectional study of developing resting masseter activity in different Angle classifications in adolescence. Cranio. 2009; 27(1):39-45.

12. Sforza C, Peretta R, Grandi G, Ferronato G, Ferrario VF. Soft tissue facial planes and masticatory muscle function in skeletal Class III patients before and after orthognathic surgery treatment. $\mathrm{J}$ Oral Maxillofac Surg. 2008; 66(4):691-8.

13. Tecco S, Caputi S, Festa F. Electromyographic activity of masticatory, neck and trunk muscles of subjects with different skeletal facial morphology: a cross-sectional evaluation. J Oral Rehabil. 2007; 34(7):478-86.

14. Tanaka K, Yamaguchi K, Tamura H, Ichida T. Changes of chewing movement before and after orthognathic surgical treatment in skeletal Class III patients. J Kyushu Dental Soc. 2007; 60(6):179-90. 15. Yamada EK, Siqueira KO, Xerez D, Koch HA, Costa MMB. A influência das fases oral e faríngea na dinâmica da deglutição. Arq Gastroenterol. 2004; 41(1):18-23.

16. Cleall JF. Deglutition: a study of form and function. Am J Orthod. 1965; 51(8):566-94.

17. Williams PL, Warwick R, Dyson M, Bannister LH. Gray anatomia 37.ed. Rio de Janeiro: Guanabara Koogan; 1995. 1252p. 
18. CraryMA, CarnabyGD, GroherME.Biomechanical correlates of surface electromyography signals obtained during swallowing by healthy adults. J Speech Lang Hear Res. 2006; 49(1):186-93.

19. Miralles R, Gutiérrez C, Zucchino G, Cavada G, Carvajal R, Valenzuela S, et al. Body position and jaw posture effects on supra- and infrahyoid electromyographic activity in humans. Cranio. 2006; 24(2):98-103.

20. Junqueira PS, Campiotto AR. A investigação da mastigação em indivíduos portadores de Classe III de Angle. Pró-Fono. 1992; 4(2):29-31.

21. Moreno I, Sánchez T, Ardizone I, Aneiros $\mathrm{F}$, Celemin A. Electromyographic comparisons between clenching, swallowing and chewing in jaw muscles with varying occlusal parameters. Med Oral Patol Oral Cir Bucal. 2008; 13(3):E207-13.

22. Jardini RS, Ruiz LS, Moysés MA. Electromyographic analysis of the masseter and buccinator muscles with the Pró-Fono facial exerciser use in bruxers. Cranio. 2006; 24(1):29-37. 23. Dominguez GC. Nova visão em ortodontia e ortopedia funcional dos maxilares. In: Bérzin $F$, Nagae MH. Memória muscular e recidivas. São Paulo: Santos; 2006. p. 425-31.

24. Eggensperger N, Smolka W, Rahal A, Ilzuka $\mathrm{T}$. Skeletal relapse after mandibular advancement and setback in single-jaw surgery. J Oral Maxillofac Surg. 2004; 62(12):1486-96.

25. Sgobbi de Faria CR, Bérzin F. Electromyographic study of the temporal, masseter and suprahyoid muscles in the mandibular rest position. $\mathrm{J}$ Oral Rehabil. 1998; 25(10):776-80.

26. de Mayo T, Miralles R, Barrero D, Bulboa A, Carvajal D, Valenzuela $S$, et al. Breathing type and body position effects on sternocleidomastoid and suprahyoid EMG activity. J Oral Rehabil. 2005; 32(7):487-94.

27. Fresno MJ, Miralles R, Valdivia J, Fuentes A, Valenzuela S, Ravera MJ, et al. Electromyographic evaluation of anterior temporal and suprahyoid muscles using habitual methods to determine clinical rest position. Cranio. 2007; 25(4):257-63.

RECEBIDO EM: 24/04/2009

ACEITO EM: 28/09/2009

Endereço para correspondência:

Mirian Hideko Nagae

Av. Paulista, 1195 ap. 152

São Paulo - SP

CEP: 014900-100

E-mail: mnagae@uol.com.br /

mnagae@fcm.unicamp.br
28. Rainold A, Minetto MA. International Society of Electrophysiology and Kinesiology (ISEK). Proceedings of the XVIth Congress International Society of Electrophysiology and Kinesiology; 2006. Centro di Bioingegneria Politecnico di Torino: Torino, Italy.

29. Cram J, Kasman GS, Holtz J. Introduction to surface electromyography. Maryland: Gaithersburg, Aspen Publication; 1998. 244p.

30. De Luca CJ. Wartenweiler Conferência Comemorativa. [homepage na internet] Centro e Departamento de Pesquisa de Engenharia Biomédica Neuromuscular e Departamento de Neurologia da Universidade de Boston, 1993. Direitos autorais por Delsys Inc. 1997 [acesso em 29 maio 2009] Disponível em: URL: http://www. delsys.com

31. Logemann JA. Evaluation and treatment of swallowing disorders. Austin: Pro-ed; 1983.

32. Yang JF, Winter DA. Electromyographic amplitude normalization methods: improving their sensitivity as diagnostic tools in gait analysis. Arch Phys Med Rehabil. 1984; 65(9):517-21.

33. Jackson JA, Mathiassen SE, Dempsev PG. Methodological variance associated with normalization of occupational upper trapezius EMG using sub-maximal reference contractions. J Electromyogr Kinesiol. 2009; 19(3):416-27.

34. Morris AD, Kemp GJ, Lees A, Frostick SP. A study of the reproducibility of three different normalisation methods in intramuscular dual fine wire electromyography of the shoulder. J Electromyogr Kinesiol. 1998; 8(5):317-22.

35. Ervilha UF, Duarte M, Amadio AC. Estudo sobre procedimentos de normalização do sinal eletromiográfico durante o movimento humano. Rev Bras Fisiot. 1998; 3(1):15-20.

36. System Analysis Statistical SAS Institute Inc. The SAS System, Release 8.2 SAS Institute Inc. Cary: NC; 1991.

37. De Sá FPG. As bases fisiológicas da ortopedia maxilar. São Paulo: Santos; 1994. 40p.

38. Ash MM, Ramfjord S. Oclusão. 4. ed. Rio de Janeiro: Guanabara Koogan; 1995.

39. Nakamura Y, Katakura N. Generation of masticatory rhythm in the brainstem. Neuroscie Res. 1995; 23(1):1-19. 